\title{
Maintenance of remission with low-dose olopatadine hydrochloride for itch in well- controlled chronic urticaria
}

This article was published in the following Dove Press journal:

Clinical, Cosmetic and Investigational Dermatology

18 September 2012

Number of times this article has been viewed

\author{
Teruhiko Makino' \\ Yoshiaki Takegami' \\ Mati Ur Rehman' \\ Yoko Yoshihisa' \\ Waka Ishida ${ }^{2}$ \\ Takashi Toyomoto ${ }^{3}$ \\ Tadamichi Shimizu' \\ 'Department of Dermatology, \\ University of Toyama, Toyama, Japan; \\ 2Department of Dermatology, \\ Niigata Central Hospital, Joetsu, \\ Japan; ${ }^{3}$ Department of Dermatology, \\ Saiseikai Takaoka Hospital, \\ Takaoka, Japan
}

Background: The long-term follow-up of chronic urticaria (CU) is important to ensure the adequate treatment of patients. Olopatadine hydrochloride is one of the second-generation nonsedating antihistamines.

Methods: This study was designed to assess the optimal dose of olopatadine to suppress symptoms of chronic urticarial itch in well-controlled patients. After CU patients were treated with $10 \mathrm{mg}$ olopatadine, patients having a visual analog scale (VAS) itch score of less than 20 were randomly allocated into one of three groups: $10 \mathrm{mg} /$ day $(\mathrm{n}=35), 5 \mathrm{mg} / \mathrm{day}(\mathrm{n}=30)$, or no medication $(\mathrm{n}=32)$.

Results: The suppressive effects of both the $5 \mathrm{mg}$ and $10 \mathrm{mg}$ olopatadine treatments on the VAS itch score were more significant and longer lasting over a period of 4 weeks than the no-medication treatment. Both the 5-mg group and the 10-mg group showed improved urticarial symptoms and maintained their VAS itch score within normal limits compared to the no-medication group. The differences between the 5-mg and 10-mg groups were not significant.

Conclusion: These results demonstrate that treatment with olopatadine at a dose of $5 \mathrm{mg}$ once daily is effective and safe for the management and prevention of CU symptoms for itch in well-controlled patients.

Keywords: chronic urticaria, olopatadine, dose, antihistamine, itch, histamine

\section{Introduction}

Chronic urticaria $(\mathrm{CU})$ is characterized by the recurrence of transitory cutaneous wheals accompanied by redness, itching, and frequently angioedema, with each episode lasting longer than six weeks. ${ }^{1}$ It is known to cause severe disability and impair a person's quality of life, due to the interruption of sleep, fatigue, social isolation, energy loss, and emotional/sexual difficulties. ${ }^{2}$ Although several causes of CU have been reported, the exact cause of this disease is still unknown and regarded as idiopathic, affecting about $0.5 \%-1.0 \%$ of subjects across different populations. ${ }^{3-5}$ Previous studies have reported the natural history of $\mathrm{CU}$ in adults, and have shown that the disease goes into remission in $30 \%-55 \%$ of patients within 12 months, but in some patients the disease can be active for several years. ${ }^{6,7}$

Oral $\mathrm{H}_{1}$ antihistamines are considered to be the first-line treatment for $\mathrm{CU}^{8}{ }^{\text {but }}$ some first generations of these agents cause anticholinergic and central nervous system (CNS) side effects, especially drowsiness and sedation. These are considered to be one of the major problems in treating CU patients, because these symptoms often force them to quit taking their medication, which may negatively impact patient quality of life. ${ }^{9}$ Therefore, in the European Academy of Allergy and Clinical Immu-
Correspondence: Tadamichi Shimizu Sciences, University of Toyama,

Sugitani, Toyama, Japan

Tel +8I 764347305

Fax +8I 764345028

Email shimizut@med.u-toyama.ac.jp 
nology/Global Allergy and Asthma European Network/ European Dermatology Forum/World Allergy Organization guidelines, nonsedating antihistamines are recommended for the treatment of patients with CU. Second-generation antihistamines are commonly thought to have reduced CNS side effects.

Olopatadine hydrochloride is one of the second-generation nonsedating antihistamines, and it is used to treat allergic disorders such as urticaria, rhinitis, and atopic dermatitis in Japan. ${ }^{10}$ It also has an inhibitory effect on the release of inflammatory lipid mediators, such as leukotriene and thromboxane, from human polymorphonuclear leukocytes and eosinophils. ${ }^{11}$ It was proven to be more potent and stable with regard to its antihistaminic effects in skin responses compared to other commonly used second-generation antihistamines in a double-blind, randomized controlled study. ${ }^{12}$ However, olopatadine hydrochloride has been reported to induce CNS side effects, despite being a second-generation antihistamine. ${ }^{13}$

It has been reported that a double dose of the prescribed drug or a change of drug was recommended for the treatment of patients with urticaria refractory to the standard dose of antihistamines. ${ }^{14}$ However, there have been no published reports regarding treatment strategy for well-controlled CU patients, including whether patients should be treated with the standard dose, reduced to half the dose, or whether the medication should be stopped. This study was conducted to compare the effects of olopatadine hydrochloride (10 $\mathrm{mg}, 5 \mathrm{mg}$, and $0 \mathrm{mg}$ /day) for itch in well-controlled CU patients to investigate the optimal dose of olopatadine that would be convenient for $\mathrm{CU}$ patients.

\section{Materials and methods \\ Patients}

Patients with CU symptoms for at least 6 weeks without any identifiable cause attending our clinic between April 2009 and March 2011 were recruited in this study. Physical urticaria patients, pregnant females, and lactating mothers were excluded. In addition to $\mathrm{CU}$, other allergic symptoms, including asthma, rhinitis, and conjunctivitis, were present in these patients (Table 1). All patients gave written informed consent for participation, which complied with all of the principles of the Declaration of Helsinki. This study was approved by the Medical Ethics Committees of the University of Toyama, Toyama, Japan.

\section{Study design}

The study was performed in a randomized, open manner. The study design is shown in Figure 1. A visual analog scale (VAS) itch score was used to evaluate the therapeutic effect of olopatadine hydrochloride. ${ }^{15}$ The scale ranges from 0 to 100, 0 indicating no itch and 100 indicating the most severe itch. The VAS itch score before and after standard treatment with $10 \mathrm{mg}$ olopatadine hydrochloride was assessed by qualified dermatologists. Thereafter, all patients noted recorded their VAS itch score at home by themselves during the study period. At first, all CU patients with a VAS itch score higher than 50 were treated with $10 \mathrm{mg}$ olopatadine hydrochloride daily for 4-6 weeks. Of these, patients having a VAS itch score less than 20 were randomly allocated into one of three groups: patients in group 1 were treated with $10 \mathrm{mg}$ olopatadine hydrochloride daily, patients in group 2 were treated with $5 \mathrm{mg}$ olopatadine hydrochloride daily, and patients in group 3 were stopped taking medication. This study was performed for 4 weeks. During this period, patients were not allowed to take any other antihistamines and corticosteroids.

\section{Time of follow-up}

The efficacy end point was defined as the length of time the VAS itch score remained less than 50 without any additional treatment. A VAS itch score exceeding 50 was considered to be an itch recurrence. These patients were dropped from the protocol and such additional treatments as increase in the olopatadine hydrochloride dose, a change in antihistamine, or additional use of corticosteroid was prescribed.

Table I Baseline characteristics of patients

\begin{tabular}{lllll}
\hline & $\mathbf{1 0} \mathbf{~} \mathbf{g}$ & $\mathbf{5 ~} \mathbf{~ g}$ & No medication & Overall \\
\hline $\mathrm{n}$ & 35 & 30 & 32 & 97 \\
Age (years), mean, & $55.2 \pm 14.9$ & $55.0 \pm 13.5$ & $59.1 \pm 15.1$ & $56.4 \pm 14.5$ \\
range & $24-79$ & $28-8 \mid$ & $26-83$ & $24-83$ \\
Sex (male/female) & $31.4 \% / 68.6 \%$ & $16.7 \% / 83.3 \%$ & $28.1 \% / 7 \mid .9 \%$ & $25.8 \% / 74.2 \%$ \\
Other allergic symptoms & $61.8 \%$ & $67.9 \%$ & $71.0 \%$ & $66.7 \%$ \\
(asthma, rhinitis, conjunctivitis) & & & & \\
\hline
\end{tabular}




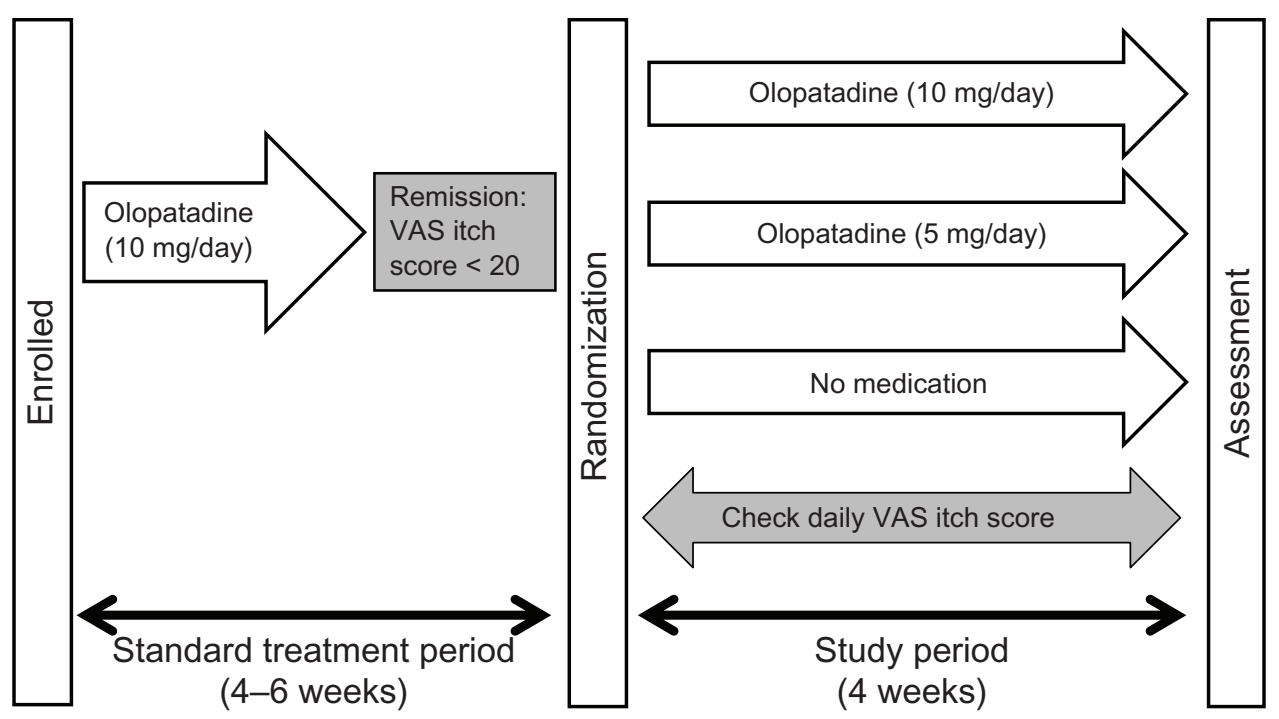

Figure I The study design.

\section{Statistical analysis}

The log-rank test was used for the analysis of the KaplanMeier curves for the time to relapse after changing the dose of olopatadine hydrochloride. All statistical calculations were performed with the SAS software program (SAS Institute, Cary, NC). The significance of differences in the remission time between the groups was evaluated by the Wilcoxon signed-rank test. Values of $P<0.05$ were considered to be statistically significant.

\section{Results}

\section{Disposition of patients}

The distribution of patients enrolled in this study is shown in Figure 2. A total of $97 \mathrm{CU}$ patients (25 male and 72 female, age range $24-83$ years, mean age 56.4 years) were evaluated in this study (10 mg, 35; $5 \mathrm{mg}, 30$; no medication, 32). Demographic and other baseline characteristics of the patients are presented in Table 1. There were no significant between-group differences in the distribution of the baseline characteristics. Before the standard treatment, the average VAS itch scores in the CU patients was 56.4, and VAS itch scores in the 10-mg, 5-mg, and no-medication group were $60.1,55.6$, and 53.5, respectively. After the standard treatment with $10 \mathrm{mg}$ olopatadine, the average VAS score decreased to 8.9, with scores decreased to $10.2,4.4$, and 12.2 in the $10-\mathrm{mg}$, 5 -mg, and no-medication groups, respectively. These findings indicated that VAS itch score was significantly decreased in the three groups after the standard treatment compared to

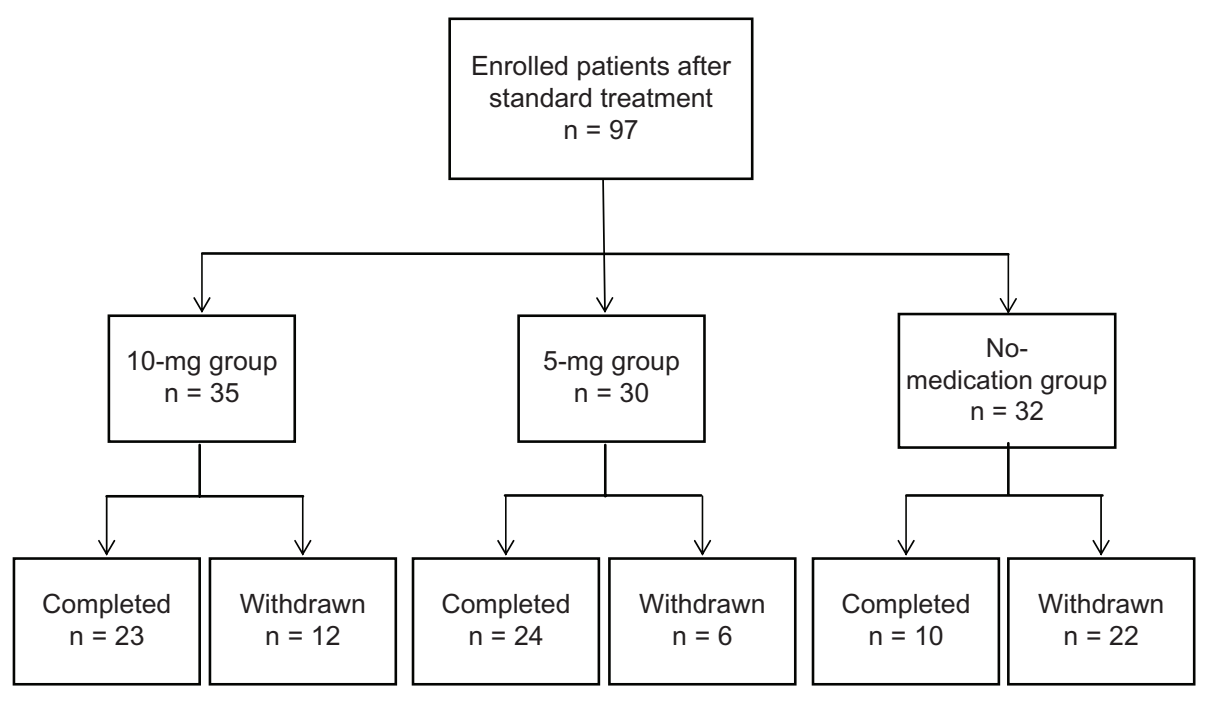

Figure 2 Disposition of the patients. 
VAS score before treatment in each group $(P<0.001)$. There were no significant differences in VAS itch score between the groups before and after the standard treatment with $10 \mathrm{mg}$ olopatadine (Figure 3).

\section{Efficacy of treatment with olopatadine hydrochloride}

An increase in VAS itch score (to more than 50) was evaluated as the end point of the study (Figure 4). In the 10-mg group, 23 of the 35 patients stayed on the study protocol during the observation period. Eight patients were observed to have a VAS score higher than 50 and discontinued their study participation. In the 5-mg group, 24 of the 30 patients completed the protocol, and five patients withdrew because their VAS score exceeded 50. In the no-medication group, only 10 of the 32 patients stayed on the study protocol during the observation period. Twenty-two patients withdrew, and the majority (19 out of 22 cases) relapsed within the first 7 days (Figure 4). No cause other than urticaria relapse was observed during the study.

The period for which the VAS itch score was maintained at less than 50 in the treatment groups was compared with that in the no-medication group (log-rank test). The period in both the 10-mg and 5-mg treatment groups was significantly longer than that in the no-medication group ( $P<0.005$ and $<0.001$, respectively). The difference between the 10-mg and 5-mg groups was not significant $(P=0.17)$.

The average remission period was 19.1 days in the $10-\mathrm{mg}$ group, 23.7 days in the 5-mg group, and 11.8 days in the no-medication group. The average remission period was

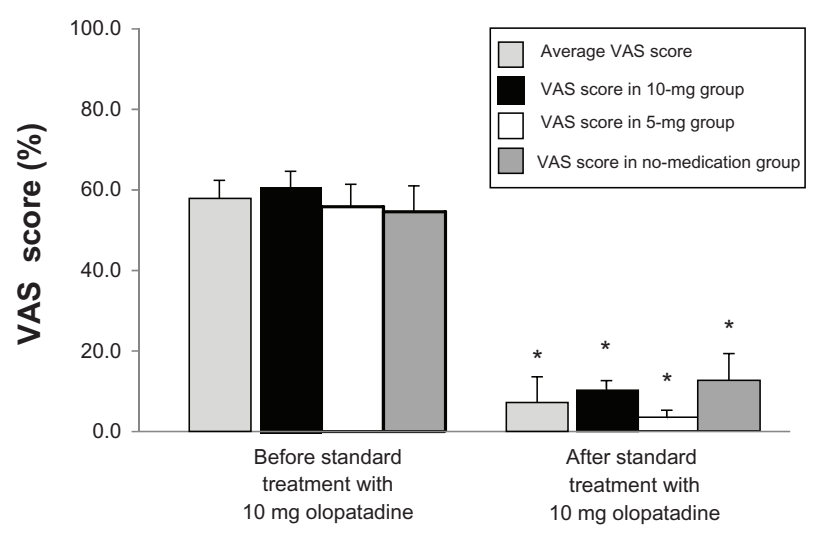

Figure 3 A comparison of the visual analog scale (VAS) itch scores in the three groups after the standard treatment with $10 \mathrm{mg}$ olopatadine hydrochloride.

Notes: The VAS itch score was more than 50 before the treatment in all the groups, and it decreased to less than 20 in all of the groups after the treatment. There were no significant differences observed between the groups before and after treatment with $10 \mathrm{mg}$ olopatadine. $* P<0.001$ compared to the VAS score before treatment with $10 \mathrm{mg}$ olopatadine in each group. also compared for the three groups. The average period in the 10-mg and 5-mg groups was significantly longer than that in the no-medication group $(10 \mathrm{mg}, P<0.01 ; 5 \mathrm{mg}$, $P<0.001)$.

\section{Safety}

Treatment with olopatadine hydrochloride was well tolerated. No patients reported adverse events such as sedation and drowsiness during the study period.

\section{Discussion}

Patients with CU face significant difficulties in their daily routine, primarily due to sleep disturbance, recurrent itch, fatigue, social isolation, and emotional distress, and the impact of CU on a patient's quality of life is considered to be similar to that of a patient awaiting coronary artery bypass surgery. ${ }^{2,16}$ Although first-generation antihistamines have been found to be effective for relieving symptoms, they cause CNS side effects, such as sedation and psychomotor disturbances. ${ }^{17,18}$ Olopatadine hydrochloride is one of the nonsedating second-generation antihistamines, and is used for treating allergic disorders such as urticaria, rhinitis, and atopic dermatitis in Japan. ${ }^{10}$ Its usual dose for treatment of $\mathrm{CU}$ is $10 \mathrm{mg} /$ day. It exerts more rapid and prolonged antihistaminic effects in comparison with other second-generation antihistamines. ${ }^{19}$

The present study was designed to investigate the optimal dose of olopatadine hydrochloride to provide increased convenience for itch in well-controlled CU patients with fewer side effects. The period that the VAS itch score was maintained at less than 50 in both the 10-mg and 5-mg groups was significantly longer than that in the no-medication group. However, the difference between the 10-mg and 5-mg groups was not significant. The average remission period in both the 10-mg and 5-mg groups was also significantly longer than that in the no-medication group. These results demonstrated that treatment with $5 \mathrm{mg}$ olopatadine hydrochloride was as effective as $10 \mathrm{mg}$ olopatadine hydrochloride for controlling symptoms in CU patients. In addition, the present study also indicates that $\mathrm{CU}$ patients can be kept on medication at either $5 \mathrm{mg}$ or $10 \mathrm{mg}$ to achieve long-term symptom improvement; however, stopping medication can lead to the development of severe symptoms associated with recurrence within a short period.

In this study, no adverse effects including drowsiness and sedation were reported by patients in any of the three groups. However, some previous studies have described that olopatadine hydrochloride induced CNS side effects, 


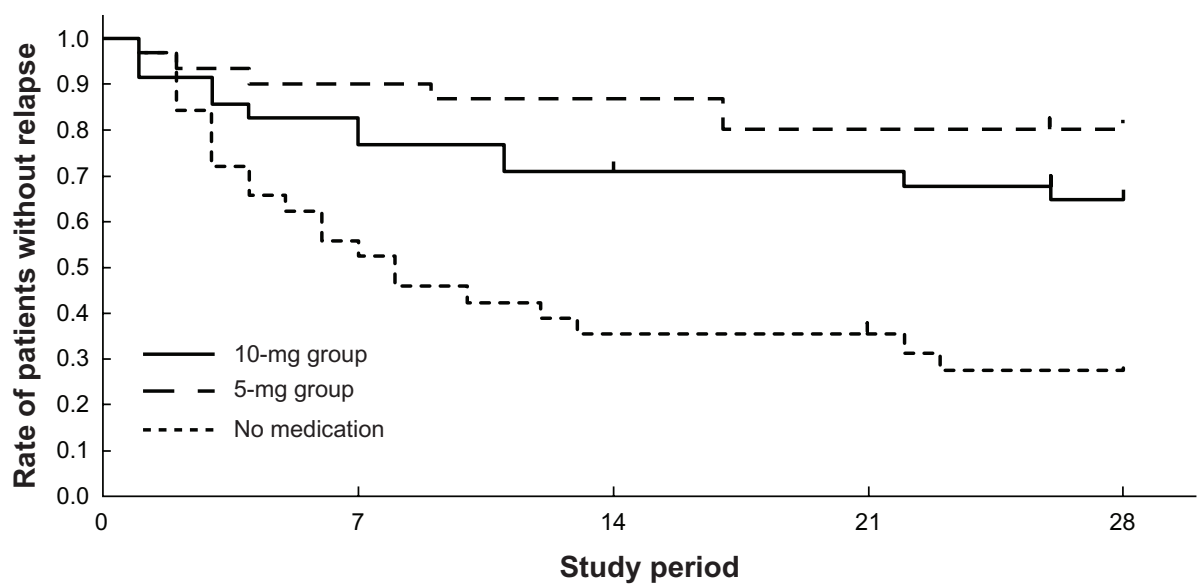

Figure 4 Rate of patients without relapse.

Notes: Visual analog scale itch score being maintained at less than 50 was significantly prolonged in the 5 -mg and 10 -mg groups compared with the no-medication group $(P<0.005$ and $P<0.00$ I, respectively, log-rank test).

in spite of the fact that it is a second-generation nonsedating antihistamine. ${ }^{13}$ Furthermore, $10 \mathrm{mg}$ olopatadine hydrochloride was also reported to cause a decrease in activity, although it did not affect psychomotor/cognitive performance. ${ }^{20}$ Therefore, we suggest that treatment with $5 \mathrm{mg}$ olopatadine hydrochloride is beneficial and provides long-lasting symptom relief for CU patients, with no or fewer CNS side effects such as drowsiness or sedation. The efficacy of $5 \mathrm{mg}$ olopatadine hydrochloride shown in our study was consistent with a previous study, in which treatment with olopatadine at $5 \mathrm{mg} /$ day caused a significant improvement in seasonal allergic symptoms in Japanese patients with cedar pollinosis, upon exposure to cedar pollen in an environmental exposure unit. $^{21}$ The fact that medications at the lowest dosing frequency improved inaccurate compliance for longterm treatment might also be supportive of our hypothesis. ${ }^{22}$ Once-daily dosing reduces the chance of missed doses and provides increased convenience for allergic patients, especially for those who feel difficulties with dosing more than once per day. ${ }^{23}$

In conclusion, this study indicates that treatment with olopatadine hydrochloride at $5 \mathrm{mg}$ once daily can maintain the improvement of urticarial symptoms the same as $10 \mathrm{mg}$ /day with fewer CNS side effects and appears to be safe and effective for the management and prevention of CU.

\section{Disclosure}

The authors have no conflicts of interest to declare.

\section{References}

1. Morita K, Koga T, Moroi Y, Urabe K, Furue M. Rapid effects of olopatadine hydrochloride on the histamine-induced skin responses. J Dermatol. 2002;29:709-712.
2. O’Donnell BF, Lawlor F, Simpson J, Morgan M, Greaves MW. The impact of chronic urticaria on the quality of life. Br J Dermatol. 1997; 136:197-201.

3. Kozel MM, Sabroe RA. Chronic urticaria: aetiology, management and current and future treatment options. Drugs. 2004;64:2515-2536.

4. Greaves MW. Chronic idiopathic urticaria. Curr Opin Allergy Clin Immunol. 2003;3:363-368.

5. Powell RJ, Du Toit GL, Siddique N, et al. BSACI guidelines for the management of chronic urticaria and angio-oedema. Clin Exp Allergy. 2007;37:631-650.

6. Kozel MM, Mekkes JR, Bossuyt PM, Bos JD. Natural course of physical and chronic urticaria and angioedema in 220 patients. $J$ Am Acad Dermatol. 2001;45:387-391.

7. Van der Vafk PG, Moret G, Kiemeney LA. The natural history of chronic urticaria and angioedema in patients visiting a tertiary referral centre, Br J Dermatol. 2002;146:110-113.

8. Monroe E. Review of $\mathrm{H} 1$ antihistamines in the treatment of chronic idiopathic urticaria. Cutis. 2005;76:118-126.

9. Belsito DV. Second-generation antihistamines for the treatment of chronic idiopathic urticaria. J Drugs Dermatol. 2010;9:503-512.

10. Ohmori K, Hayashi K, Kaise T, et al. Pharmacological, pharmacokinetic and clinical properties of olopatadine hydrochloride: a new antiallergic drug. Jpn J Pharmacol. 2002;88:379-397.

11. Ohmori K, Hasegawa K, Tamura T, et al. Properties of olopatadine hydrochloride, a new antiallergic/antihistaminic drug. Arzneimittelforschung. 2004;54:809-829.

12. Takahashi H, Zhang Y, Morita E. Evaluation of the antihistamine effects of olopatadine, cetirizine and fexofenadine during a $24 \mathrm{~h}$ period: a double-blind, randomized, crossover, placebo-controlled comparison in skin responses induced by histamine iontophoresis. Arch Dermatol Res. 2008:300:291-295.

13. Takahashi H, Ishida-Yamamoto A, Iizuka H. Effects of bepotastine, cetirizine, fexofenadine, and olopatadine on histamine-induced whealand flare-response, sedation, and psychomotor performance. Clin Exp Dermatol. 2004;29:526-532.

14. Okubo Y, Shigoka Y, Yamazaki M, Tsuboi R. Double dose of cetirizine hydrochloride is effective for patients with urticaria resistant: a prospective, randomized, non-blinded, comparative clinical study and assessment of quality of life. J Dermatolog Treat. Epub August 24, 2011.

15. Grattan CE, O’Donnell BF, Francis DM, et al. Randomized double-blind study of cyclosporin in chronic 'idiopathic' urticaria. Br J Dermatol. 2000;143:365-372.

16. Staubach P, Eckhardt-Henn A, Dechene M, et al. Quality of life in patients with chronic urticaria is differentially impaired and determined by psychiatric comorbidity. Br J Dermatol. 2006;154:294-298. 
17. Passalacqua G, Bousquet J, Bachert C, et al. The clinical safety of H1-receptor antagonists. An EAACI position paper. Allergy. 1996;51: $666-675$.

18. Welch MJ, Meltzer EO, Simons FE. H1-antihistamines and the central nervous system. Clin Allergy Immunol. 2002;17:337-388.

19. Kamei H, Isaji A, Noda Y, et al. Effects of single therapeutic doses of promethazine, fexofenadine and olopatadine on psychomotor function and histamine-induced wheal- and flare-responses: a randomized double-blind, placebo-controlled study in healthy volunteers. Arch Dermatol Res. 2012;304:263-272.

20. Kamei H, Noda Y, Ishikawa K, et al. Comparative study of acute effects of single doses of fexofenadine, olopatadine, d-chlorpheniramine and placebo on psychomotor function in healthy volunteers. Hum Psychopharmacol. 2003;18:611-618.
21. Enomoto T, Lu HQ, Yin M, et al. Evaluation of the efficacy and safety of olopatadine and fexofenadine compared with placebo in Japanese cedar pollinosis using an environmental exposure unit. J Investig Allergol Clin Immunol. 2009;19:299-305.

22. Eisen SA, Miller DK, Woodward RS, Spitznagel E, Przybeck TR. The effect of prescribed daily dose frequency on patient medication compliance. Arch Intern Med. 1990;150:1881-1884.

23. Guest JF, Davie AM, Ruiz FJ, Greener MJ. Switching asthma patients to a once-daily inhaled steroid improves compliance and reduces healthcare costs. Prim Care Respir J. 2005;14:88-98.

\section{Publish your work in this journal}

Clinical, Cosmetic and Investigational Dermatology is an international, peer-reviewed, open access, online journal that focuses on the latest clinical and experimental research in all aspects of skin disease and cosmetic interventions. All areas of dermatology will be covered; contributions will be welcomed from all clinicians and basic science researchers globally. This journal is indexed on CAS. The manuscript management system is completely online and includes a very quick and fair peer-review system, which is all easy to use. Visit http://www.dovepress.com/testimonials.php to read real quotes from published authors.

Submit your manuscript here: http://www.dovepress.com/clinical-cosmetic-and-investigational-dermatology-journal 